\title{
Acknowledgement to Reviewers of Journal of Imaging in 2019
}

Journal of Imaging Editorial Office

MDPI, St. Alban-Anlage 66, 4052 Basel, Switzerland

Published: 17 January 2020

The editorial team greatly appreciates the reviewers who have dedicated their considerable time and expertise to the journal's rigorous editorial process over the past 12 months, regardless of whether the papers are finally published or not. In 2019, a total of 79 papers were published in the journal, with a median time to first decision of 21 days and a median time from submission to publication of 51 days. The editors would like to express their sincere gratitude to the following reviewers for their generous contribution in 2019:

\begin{tabular}{ll} 
Abdellatif, Mohamed & Chang, Jiyul \\
Adler, Amir & Chen, Bo-Hao \\
Afonso, Manya & Chen, Chen \\
Ahmad, Muhammad & Chen, Minsi \\
Alex, Aneesh & Choi, Hojong \\
Alfalou, Ayman & Choi, Woo June \\
Aliotta, Francesco & Chuang, Yung-Yu \\
Allegra, Dario & Cinzano, Pierantonio \\
Alparone, Luciano & Clement, Yuen \\
Andreić, Željko & Coakley, Kevin J. \\
Arines, Justo & Cozot, Remi \\
Ataman, Çağlar & Cozzolino, Daniel \\
Bae, Eui Won & Crocco, Lorenzo \\
Bará, Salvador & Daffara, Claudia \\
Barada, Daisuke & Dasygenis, Minas \\
Barentine, John C. & De Bernardi, Elisabetta \\
Barry, David J & De Sousa, Jose T. \\
Beltran, Carlos & Deng, Lei \\
Bernardes, Rui & Di Massa, Giuseppe \\
Bevacqua, Martina T. & Di Nunzio, Luca \\
Bialkowski, Konstanty & Diez, Yago \\
Blanco Heras, Dora & Durou, Jean-Denis \\
Blasinski, Henryk & Eibert, Thomas \\
Boulgouris, Nikolaos V. & Emberton, Simon \\
Bourgeois, Steve & Ezekiel, Soundararajan \\
Buch, Anders Glent & Farhad, Merchant \\
Burrascano, Pietro & Farid, Muhammad Shahid \\
Byun, Hyeran & Fernandes, Carlos \\
Cabaret, Laurent & Fernández-Caballero, Antonio \\
Calvo-Zaragoza, Jorge & Ferrández Vicente, José Manuel \\
Carlson, Lars-Anders & Fhager, Andreas \\
Carrara, Fabio & Filip, Jiri \\
Celona, Luigi & Fisher, Robert \\
& \\
\hline
\end{tabular}


Fontana, Raffaella

Frances-Villora, Jose

Gällström, Andreas

Gao, Shangce

Garrett, Joseph L.

Gennaro, Claudio

Giacomelli, Michael

Gomez-Donoso, Francisco

González-Hidalgo, Manuel

Goree, John

Graff, Marie

Grammalidis, Nikos

Grana, Costantino

Grosse, Mirco

Guerrero, Paul

Hadar, Ofer

Hanly, Joseph J.

Hatt, Mathieu

He, Lifeng

Heddleston, John

Helbig, Marko

Helbin, Piotr

Hirayama, Ryuji

Hoppe, Adam

Hung, Li-Wei

Huynh-The, Thien

Imiya, Atsushi

Ipek, Özlem

Itrić, Katarina

Jaworska, Tatiana

Jeffrey, Ian

Jørgensen, Jakob Sauer

Joslin, Chris

Juan, Guillermo

Jung, Ki-Hyun

Kelly, Christopher

Keszöcze, Oliver

Khatibi, Siamak

Khmaladze, Alexander T.

Killer, Carsten

Kim, Kyungsang

Kirchner, Thomas

Knapek, Christina

Kocifaj, Miroslav

Kolláth, Zoltán

Konda, Pavan

Kronreif, Gernot

Kurrant, Doug

Kyba, Christopher

Le Pendu, Mikael

Lee, Gueesang

Lee, Sang-Heon
Lee, Soochahn

Leeser, Miriam

Leone, Giovanni

Li, Xiujun

Lima, Raul Cerveira

López-Quintero, Manuel Ignacio

Mainprize, James

Mandal, Subhamoy

Marita, Tiberiu

Martin, Arnaud

Martin, Iain

Martins, Gaby

Massa, Andrea

Massanet, Sebastia

Matthews, Lorin

Mayerich, David

Messelodi, Stefano

Meyer-Baese, Anke

Milej, Daniel

Miyata, Takamichi

Molinara, Mario

Mondal, Suman B

Morrow, Ivor

Moseev, Dmitry

Mutschler, Christopher

Nagata, Fusaomi

Nayak, Rohit

Netzel, Henryka

Nevin, Austin

Newson, Alasdair

Nguyen, Binh

Nguyen, Thanh

Nguyen, Tuan T.

Nomura, Takanori

Nunes, Paulo

Nuutinen, Mikko

Oikawa, Kenichi

Ortolani, Sergio

Pagliarulo, Vito

Panáková, Daniela

Park, Jaehwa

Pavone, Santi Concetto

Pedersoli, Marco

Pepe, Francesco V.

Perra, Cristian

Perri, Stefania

Piana, Michele

Pirri, Fiora

Pont, Sylvia

Popov, Vladimir

Pradeep, Buddharaju

Prasath, Surya 
Presotto, Luca

Putnam, Andrew

Qi, Guanqiu

Queau, Yvain

Radonic, Vasa

Raffaeli, Roberto

Rai, Robba

Ramchandani, Divya

Randazzo, Andrea

Rasti, Pejman

Reiser, David

Rezazadegan Tavakoli, Hamed

Ribas, Salvador J.

Rodríguez, Airam

Rohrbach, Anna

Roig, Bernardino

Romeo, Stefania

Royle, Stephen

Saeed, Khalid

Safonov, Ilia

Salewski, Mirko

Salido, Jesús

Sanchez, Alejandro

Saur, Günter

Savino, Alessandro

Sawall, Stefan

Schabowicz, Krzysztof

Schiopu, Ionut

Sesma-Sara, Mikel

Sharma, Harshita

Shen, Yaochun

Shi, Jie

Simon-Chane, Camille

Skalski, Andrzej

Solis Alfaro, Jorge

Soltaninejad, Mohammadreza

Soria, Xavier

Stevenson, Gordon

Strokina, Nataliya

Strömberg, Tomas

Tahmassebi, Amirhessam
Takahashi, Tomohiro

Tankam, Patrice

Tarasov, Andrei

Ten Bosch, L.F.M.

Theodorakeas, Panagiotis

Thoma, Markus

Tiberi, Gianluigi

Ticos, Cătălin M.

Timmins, Claire

Tobon, Jorge

Tsiklauri, David

Tsinos, Christos G.

Tumurbaatar, Tserennadmid

Turkington, Alice

Ubaru, Shashanka

Ukwatta, Eranga

Upneja, Rahul

Vallez, Noelia

Van Eijnatten, Maureen

Venkataraman, Rajesh

Vozel, Benoit

Wang, Kang

Wang, Qian

Williams, Jeremiah

Woods, Roger

Yasukawa, Shinsuke

Yeom, Jiwoon

Yoon, Jonghee

Yu, Hui

Yu, Weimiao

$\mathrm{Yu}, \mathrm{Xin}$

Zanetti, Massimo

Zeng, Kai

Zhang, Heye

Zhang, Yu

Zhong, Yu

Zhou, Jun

Zhurov, Alexei I.

Zilberti, Luca

Zolliker, Peter 